\title{
DECODE: Exploiting Shadow Fading to DEtect COMoving Wireless DEvices
}

\author{
Gayathri Chandrasekaran, Mesut Ali Ergin, Marco Gruteser, Member, IEEE Computer Society, \\ Richard P. Martin, Jie Yang, and Yingying Chen
}

\begin{abstract}
We present the DECODE technique to determine whether a set of transmitters are comoving, i.e., moving together in close proximity. Comovement information can find use in applications ranging from inventory tracking to social network sensing and to optimizing mobile device localization. The positioning errors from indoor RSS-based localization systems tend to be too large, making it difficult to detect whether two devices are moving together based on the interdevice distances. DECODE achieves accurate comovement detection by exploiting the correlations in positioning errors over time. DECODE can not only be implemented in the position space but also in the signal space where a correlation in shadow fading due to objects blocking the path between the transmitter and receiver exists. This technique requires no change in or cooperation from the tracked devices other than sporadic transmission of packets. Using experiments from an office environment, we show that DECODE can achieve near-perfect comovement detection at walking speed mobility using correlation coefficients computed over approximately 60-second time intervals. We further show that DECODE is generic and could accomplish detection for mixed mobile transmitters of different technologies (IEEE $802.11 \mathrm{~b} / \mathrm{g}$ and IEEE 802.15.4), and our results are not very sensitive to the frequency at which transmitters communicate.
\end{abstract}

Index Terms-Comovement, localization, Received Signal Strength Indicator (RSSI), shadow fading, correlation coefficient.

\section{INTRODUCTION}

$\mathrm{M}$ ANY location-aware applications benefit from higherlevel information about the movements of transmitters. One instance of such higher-level information is comovement, which describes whether a set of transmitters is moving together on a common path. Comovement information could be used to infer containment relationships, indicating, for example, that two devices are owned and carried by the same person, or that several tagged objects are placed on the same pallet. It could also be used to infer social relationships if the transmitters are carried by different persons or for optimizing localization system performance.

While it is straightforward to derive comovement relationship from position coordinates and trajectories generated by a localization system, sufficiently accurate and precise data are not always available. Indeed, our evaluation of a Bayesian WiFi localization system (M1) [1] shows that the location estimation errors lead to bias and variance in the euclidean distance between two comoving transmitters, making detection of comovement difficult. Global Positioning System (GPS) accuracy is also frequently

- G. Chandrasekaran, M.A. Ergin, and M. Gruteser are with the Wireless Information Networks Laboratory (WINLAB), Technology Centre of New Jersey, Rutgers, The State University of New Jersey, 671 Route 1 South, North Brunswick, NJ 08902-3390.

E-mail: chandrga@cs.rutgers.edu, \{ergin, gruteser\}@winlab.rutgers.edu.

- R.P. Martin is with the Department of Computer Science, Rutgers University, Hill Center, 110 Frelinghuysen Rd. Piscataway, NJ 088548019. E-mail:rmartin@cs.rutgers.edu.

- J. Yang and Y. Chen are with the Department of Electrical and Computer Engineering, Stevens Institute of Technology, Hoboken, NJ 07030.

E-mail: jyang@stevens.edu.

Manuscript received 21 Nov. 2008; revised 29 Apr. 2009; accepted 25 June 2009; published online 28 July 2009.

For information on obtaining reprints of this article, please send e-mail to: tmc@computer.org, and reference IEEECS Log Number TMC-2008-11-0468. Digital Object Identifier no. 10.1109/TMC.2009.131.

$1536-1233 / 09 / \$ 25.00$ (C) 2009 IEEE degraded in urban canyons [2], and even if signals are available, GPS receivers are not commonly used in portable devices due to their high energy consumption. For indoor environments, localization systems require the presence of multiple landmarks or receivers, which adds infrastructure cost. Coarse comovement information can also be obtained from connectivity through short-range radios [3]. This, however, requires tracking software to be installed on all mobile devices; it cannot easily be inferred through infrastructure solutions alone.

\subsection{Overview of DECODE}

In this paper, we propose the DECODE technique which detects comovement through correlated signal variations over time rather than directly measuring the signal difference between two transmitters. The technique can either work in signal space, using Received Signal Strength Indicator (RSSI) values, or in position space, using location coordinates derived from the signals.

DECODE can exploit commonalities in signal power variations, because certain fading patterns of comoving transmitters are similar. The wireless communications literature [4] distinguishes shadow and multipath fading effects that attenuate or amplify a signal in addition to the path loss due to communication distance. Shadow fading refers to obstacles in the environment that attenuate the transmitted signal when it travels through the object. The magnitude of this effect depends on the material and width of the object (e.g., about $10 \mathrm{~dB}$ attenuation was observed when an outside antenna was moved inside of a vehicle [5]). Multipath fading describes the effect that objects in the environment reflect and scatter the transmitted signal, so that the signal often arrives at the receiver along multiple paths. The signal components constructively or destructively interfere, leading to fast changes in received signal strength if the position of the receiver changes by merely 
one-half the wavelength of the communication frequency used (about $59 \mathrm{~mm}$ for ISM Band $2.4 \mathrm{GHz}$ [6] can result in signal strength changes exceeding $20 \mathrm{~dB}$ ). As transmitters or receivers move, the time-varying attenuation due to these effects will be unique for each path in space. Two receivers comoving with a separation of less than one-half wavelength can be trivially detected because they will experience nearly identical signal power curves (assuming same transmission power and antennas). For high communication frequencies in the unlicensed band, however, only few transmitters will be sufficiently close to allow such straightforward detection. Thus comovement detection has to allow significant difference in signals due to multipath fading.

Thus, this paper presents the DECODE technique, which detects comoving transmitters by correlated signal changes introduced by the shadow fading component in measured signals. While the multipath component of the signal differs, transmitters separated less than a few meters will often still observe commonalities in shadow fading since larger objects in the environment tend to block all direct signal paths to the colocated transmitters. To isolate the shadow fading component, DECODE first extracts periods of high signal variance from the observed signal strength traces over time. When operating directly in signal space, DECODE removes high-frequency multipath components of the signal and calculates a correlation coefficient over the filtered signal. A high correlation coefficient indicates comovement of the transmitters. When operating in location space, it calculates correlation over a time-series trace of coordinates reported by a localization algorithm. Localization algorithms typically average signals over time, and thus, also largely filter out multipath effects. Shadow fading can manifest itself as errors in the localization output, which DECODE can exploit. One key advantage of applying DECODE in signal space is that, in typical indoor or urban outdoor environments where shadow fading exists [7], DECODE requires only one receiver to detect comovement, while localization systems require signal measurements from multiple receivers.

\subsection{Uses of Comovement Information}

Many applications can benefit from comovement information. Some of the important ones are:

- Mapping Devices to Persons: Many location-aware applications such as Friend finders are tracking devices that act as a proxy to infer the position of the device owner. The proliferation of mobile devices and distinct radio technologies on each mobile device make monitoring this mapping of devices to their owners increasingly cumbersome. For example, as a mobile device moves from an outdoor to an inbuilding location, it may be tracked by a variety of different technologies each using a different device identifier (usually a radio MAC address). By monitoring comovement of different transmitters, a localization system may be able to infer which devices belong to the same owner, or which addresses represent the same device.

- Social Network Mining: Recent work [3] has sought to infer social relationships from mobile device connectivity patterns. Applications for such techniques include automatically determining access control policies and viral marketing. Current techniques monitor Bluetooth advertisement messages to determine when and how long devices from different owners meet. This requires software on mobile devices. The comovement techniques could also extract this information through external observations (from a communications base station).

- Localization Optimizations: Knowing that two mobile devices move together helps collaborative positioning mechanisms that provide lower energy consumption or better accuracy. For example, one device could power down its GPS receiver to conserve energy, while the other device's receiver still provides accurate position updates. In challenging environments for localization, position estimates may also be improved through redundancy.

The remainder of the paper is organized as follows: in Section 2, we review related research and Section 3 presents the DECODE technique. In Section 4, we discuss our experimental methodology and results. Section 6 discusses the advantages of the DECODE system in signal space compared to the location space and the effects of environmental mobility on DECODE's performance. Concluding remarks are given in Section 7.

\section{BACKGROUND}

\subsection{Related Work}

The previous works on detecting colocated and comoving objects have either been based on absolute location of the transmitters obtained using localization indoors and GPS outdoors or from proximity sensing using short range infrared (IR) or Bluetooth devices. We know of no other work that infers colocation or comovement directly from signal strength measurements. In this section, we classify the related work into three main categories.

Mobility detection. Several earlier studies have concentrated on distinguishing mobile and stationary transmitters. Sohn et al. [8] determine mobility from GSM traces using seven different metrics one of which is the variance in Signal Strength which is similar to our approach. Similarly, Muthukrishnan et al. [9] discuss detecting mobility from RSSI in WLAN. LOCADIO [10] again used variance to detect mobility and combined it with a two state Hidden Markov Model (HMM) to eliminate oscillations between the static and mobile states. We build on this work-detecting mobility is an integral component of the DECODE technique.

Proximity-based inference. Proximity based colocation inference techniques mainly consist of using short-range IR or Bluetooth devices to estimate distance between the transmitters. The Reality Mining project [3] [11] used Bluetooth-capable GSM phones to record the other nearby Bluetooth devices and transmit them to the central server for inferring social interaction patterns. SpotOn system [12] used radio signal attenuation to estimate the relative distance between the special tags. Though these techniques look attractive for colocation detection, they require tracking software on the devices themselves and are effective only for detecting devices that have the same technology. Our scheme is more generic as it involves measurement of RSSI which is common to GSM, WLAN, Zigbee, and Bluetooth.

Distance threshold detection. This baseline detection technique involves estimating the locations of different transmitters and deriving conclusions about comovement 
based on the distance between the estimated positions of the transmitters. Recent efforts have resulted in a plethora of methods to determine the locations of transmitters. Want et al. [13] used infrared and [14] employed ultrasound to perform localization. However, both of them required specialized infrastructure to be deployed for performing localization. On the other hand, in spite of meter-level accuracy [15], using RSS [1], [16], [17] is an attractive approach because it can reuse the existing wireless infrastructure.

RADAR [16], the first algorithm for IEEE 802.11 transmitters in this category, uses RF Fingerprint information (vector containing known locations of transmitter along with a measure of the observed signal strength at different receivers) observed at three receivers and performs a nearest neighbor matching algorithm to determine the location of the transmitters with a three meters median accuracy. Elnahrawy et al. [1] use Bayesian learning algorithm on RF fingerprints observed at three or more receivers to obtain a median 802.11 localization accuracy of 3-4 meters. The most accurate 802.11 location system to date is [18] which uses the Hidden Markov Model and Bayesian inference derived from observations at nine different receivers yielding a median accuracy of 1 meter. Further, the average localization accuracy employing RSS in a 802.15.4 (Zigbee) network [17] and an active RFID system [19] is about the same with median errors around 3-4 $\mathrm{m}$ when using four receivers.

While the recent papers [20], [21] have reported a higher accuracy localization techniques, these techniques require transmitters to perform synchronized communications which is not common across typical transmitters that we analyze in this paper. Further, these papers have not reported the accuracy in a mobile environment questioning its applicability for the detection of comovement.

Intuitively, one can derive comovement information with threshold detection on the distance between two transmitters. Compared to DECODE (in signal space), all these localization systems require three or more receivers to work in concert, whereas DECODE can be used even with only one receiver. In addition, the accuracy results reported for WiFi localization raise questions about the precision of such a detection approach. We will further address this question in the next section.

\subsection{Accuracy of Distance Threshold Detection}

If current indoor localization systems can provide sufficiently accurate location coordinates, one would detect comovement based on the distance between the two transmitters remaining below a threshold of a few meters. To verify this intuition, we conduct an experiment in an office environment with coordinates reported by a WiFi localization system using the RSS bayesian localizer M1 [1]. M1 is a lateration-based Bayesian algorithm which encodes the relationship between the RSS and 2D cartesian location coordinates using a simple log-distance propagation model. Using a training set (a vector of RSS for different known $(x, y)$ locations), M1 determines the propagation parameters for each of the receivers. It then derives the joint probability density of $(x, y)$ as a function of the observed RSS for the point to be localized and uses the mean of the derived pdf to estimate the unknown location $(x, y)$. M1 has been shown to provide qualitatively comparable accuracy to current state-of-the-art WiFi localization algorithms [1].

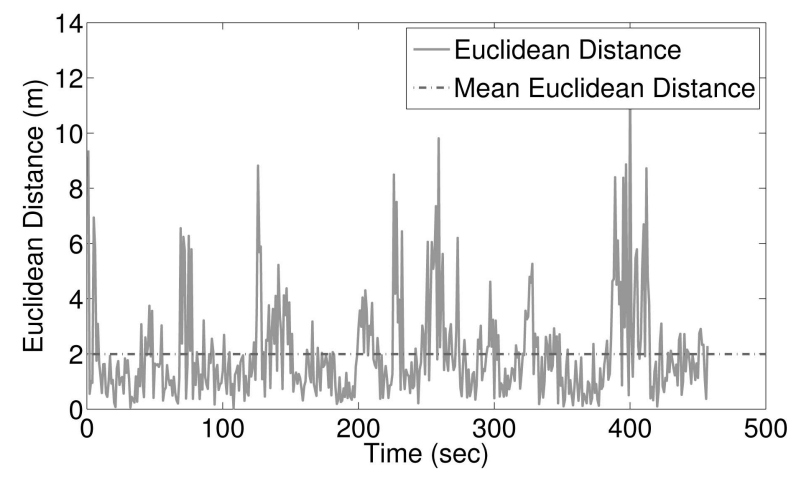

Fig. 1. The euclidean distance between the localized $(X, Y)$ positions for a comoving transmitter pair.

In our experimental setup, two IEEE 802.11g (WiFi) transmitters send 10 packets per second on the same channel, while moving together with about 6 inch separation within the office space at a constant speed of $1 \mathrm{~m} / \mathrm{sec}$. Four receivers recorded the observed Received Signal Strength (RSS) from this transmitter pair. We localize these comoving pair of transmitters in the $85 \mathrm{~m} \times 50 \mathrm{~m}$ cubicle office environment using M1 every second, based on the average RSS reported over the last second. More details on the testbed setup are provided in Section 4.1.

Fig. 1 plots the euclidean distance in geographic space between the localized points for the pair of comoving devices over time. We can see that the distance varies between 0.3 and $12 \mathrm{~m}$ with a mean distance of $2 \mathrm{~m}$. This high variance in the euclidean distance can be attributed to the following causes:

1. Typical RSS-based localization algorithms exhibit a relatively high median localization error of $3 \mathrm{~m}$ even under no mobility. This localization inaccuracy can increase with mobility thereby resulting in high distance variance.

2. The Localization algorithm estimates $X$ and $Y$ for every transmitter independently before the euclidean distance metric combines the estimated $X$ and $Y$ from each transmitter. It is possible that errors add up temporarily. It is also possible that a bias in the estimated values for one of the parameters could result in continuously high euclidean distance estimates.

These high distance errors suggest that the distance threshold detection approach cannot accurately determine comoving transmitters. This further motivates the DECODE technique, which we will describe next.

\section{Decode System Design}

The environment in which wireless communication takes place affects the received signal power (or signal-to-noise ratio). The key idea underlying the DECODE technique is exploiting shadow fading, i.e., signal attenuation due to objects blocking the path of communication. Two transmitters in close proximity will be similarly affected by surrounding buildings, furniture, or passing people. Therefore, the observed signal power from these transmitters should be correlated. This similarity in signal strength, in turn, should also translate to correlations in localization errors. 
DECODE captures these similarities by calculating the correlation coefficient over a time-series trace of signal strength or location coordinate values. The correlation coefficient measures the strength of a linear relationship between two random variables. Thus, the correlation coefficient captures similarities in the changes of two values, even if the absolute values are different. DECODE uses the Pearson's product moment correlation coefficient [22], a preferred method for quantitative measures such as the RSSI traces used. For comparison, we also evaluated another measure of correlation, Spearman's Rank correlation coefficient [23]. Unless otherwise mentioned, correlation coefficient will refer to Pearson's product moment correlation coefficient $r_{x y}$ in the remainder of this paper. For $n$ samples each from two random variables $X$ and $Y$, it is defined as

$$
r_{x y}=\frac{\sum x_{i} y_{i}-n \bar{x} \bar{y}}{(n-1) S_{x} S_{y}},
$$

where $S_{x}$ and $S_{y}$ are the sample standard deviations. The correlation coefficient lies in the interval $[-1,1]$, where 0 indicates no correlation, +1 indicates maximum positive correlation, and -1 indicates maximum negative correlation. We empirically determined a correlation coefficient threshold of 0.6 (see Section 4.4), and values that exceed this threshold indicate comovement.

Received signal strength, however, also significantly varies due to multipath fading. It can introduce received signal strength changes of more than $20 \mathrm{~dB}$ between locations separated only by half the wavelength of the carrier frequency, if no line-of-sight path to the transmitter is available. These variations render the similarities due to shadow fading difficult to detect. To address this challenge, DECODE calculates a moving average over signals, which acts as a low-pass filter to reduce or remove multipath effects.

Movement also helps detection of shadow fading similarities, because comoving transmitters will experience received signal strength changes due to shadowing at similar points in time (e.g., two comoving transmitters would pass a building corner at the same time). Intuitively, higher speed of the transmitters will increase the frequency of these changes, and thus, facilitate comovement detection. Therefore, DECODE will focus on periods of high signal variance, which typically correspond to movement.

Fig. 2 illustrates the system design and key processing steps of the DECODE system, which can use received signal strength or location coordinates for estimation. Both approaches share a number of common data collection and preprocessing steps.

In both cases, the receiver measures the received signal strength for packets emitted from transmitters. It reports a transmitter identifier, signal strength, and a reception timestamp for each observation to the DECODE processing unit, usually over an existing wired network infrastructure. In our prototype, we have implemented DECODE by monitoring the RSSI values reported for each packet reception by the receiver. RSSI has been shown to be a good indicator of channel quality [24]; hence, it should provide adequate information about fading patterns. RSSI is also available across all wireless technologies, which allows measuring comovement across different transmitters. For each transmitter, DECODE first performs time alignment and interpolation to facilitate later processing in the face of missing

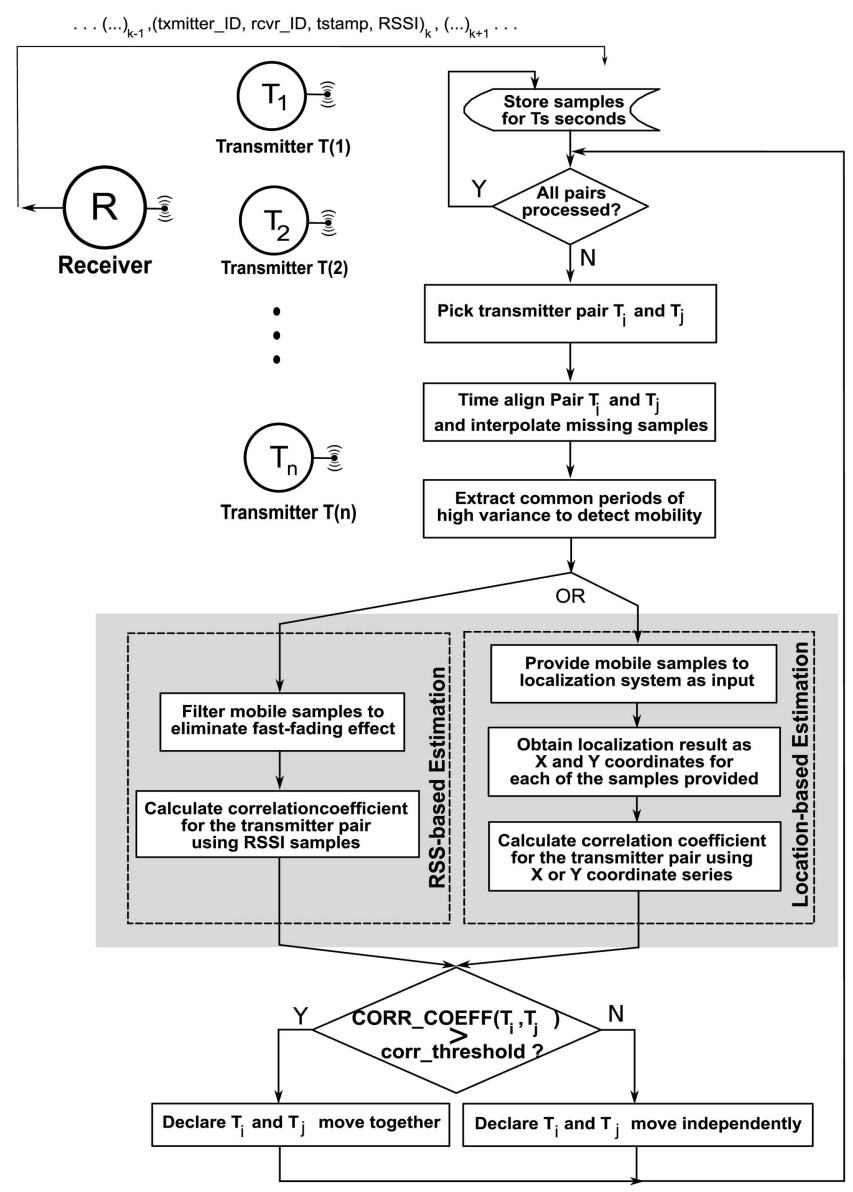

Fig. 2. System diagram and data flow.

samples. It then extracts periods of high signal variance, which are likely to correspond to movement of transmitters.

This is followed by RSS or location-specific processing steps. Finally, correlation coefficients are calculated for each transmitter pair and correlation values exceeding a specified threshold indicate comovement of a transmitter pair.

In the following sections, we give details of the common, RSS-specific, and location-specific components of DECODE.

\subsection{Common Components}

The common preprocessing steps include time alignment and extraction of high variance periods.

Time alignment. The following comovement detection seeks to compare RSSI values observed at the same time from different transmitters. The packets originating from transmitters attached to different devices may not be synchronized in time. Even if one attempts to synchronize transmitters attached to the same device, the inherent channel access delays will cause packets from these different transmitters to arrive at the receiver at slightly different times. Depending on wireless channel conditions, packets are also lost due to collisions or path loss. Thus, the time alignment step synchronizes the samples received from two transmitters. Given the packet traces for two transmitters, our implementation matches every packet from the first transmitter with the last prior packet transmission from the second transmitter. If a sample is missing from the second transmitter, this procedure 

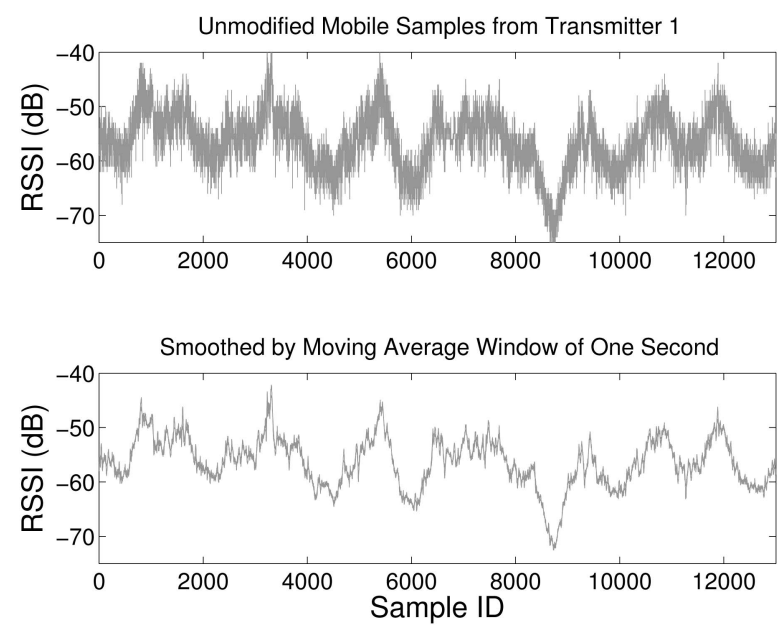

Fig. 3. Smoothing data to remove fast fading.

replaces the missing sample with the last observed sample from the second transmitter.

Extracting high variance periods. Recall that DECODE focuses on periods of mobility because during these periods, it can observe correlated signal changes due to shadow fading, and during these periods it can filter out multipath fading. Several techniques have been proposed to detect mobility [8], [9], [10], [25]. Of these, we choose the straightforward signal strength variance threshold detection technique. DECODE divides the RSSI traces into blocks. It then extracts and concatenates all blocks where the variance exceeds the specified threshold. We empirically determined the variance threshold to be three (see Section 4.6 for further discussion) and a suitable block size of 5 seconds for variance calculation.

\subsection{RSS Estimation Components}

If DECODE operates using RSS data, this is followed by filtering out multipath fading and computing correlation over RSS values.

Filtering out multipath fading. While fading is common in communication channel, the fast fading component where the signal varies in amplitude and phase over short periods of times does not contain useful information about the shadowing profile of the environment. The variance due to fast fading should thus be removed from the RSSI traces to allow calculation of correlation primarily over shadow fading components. DECODE uses a moving window averaging process with a window size of 10 packets $(1 \mathrm{sec})$. Fig. 3 shows an example of this filtering effect. Before filtering the received RSSI values vary by about $10 \mathrm{~dB}$ on time scales of less than $100 \mathrm{~ms}$. After processing, only slow variations remain, which are expected from shadow fading.

Comovement detection. The final step involves calculating correlation coefficient on the processed signal strength values from the transmitter pair under consideration. If the resulting correlation coefficient exceeds a certain threshold, we classify the transmitter pair to be comoving. We give details on determining this threshold in Section 4.

\subsection{Location Estimation Components}

The location-based estimation approach calculates the same correlation metric over time-series location coordinate data, but it requires data from several receivers to be available and a calibrated localization system. Our localization system relied on an existing signal map of the building, which discretizes spaces and contains an observed signal strength vector (each value corresponding to a different receiver) for each known location.

RSS fingerprint generation. The input to the location system is a fingerprint, an $R \times T_{s}$ matrix containing RSS values, where $R$ refers to the number of receivers (four in our setup) and $T_{s}$ to a time window in seconds. To generate these fingerprints, receivers report the transmitter identifier, signal strength, and a reception time stamp for every transmitted frame to DECODE. After generating a timealigned sample for the transmitter pairs at each of the receiver and extracting the high variance periods, the resulting RSS samples for each transmitter-receiver pair are averaged over 1-second intervals and entered into the fingerprint matrix (one matrix per transmitter). If the interval contains no observations for a specific transmitter-receiver pair, the fingerprint generator fills in a localization algorithm-specific default value of -99 .

Localization. We use a Bayesian solver [1] called M1 to perform localization. M1 is initially provided with a signal map (or training set) containing measurements from 88 different locations in $2 \mathrm{D}$ space within the building where we carried out the experiment. M1 then transforms each fingerprint matrix into a $2 \times T_{s}$ matrix of cartesian location coordinates over time, one location estimate per second.

Comovement detection. The final step involves detecting comovement from the $(X, Y)$ estimations at every second for different transmitters. To verify whether a pair of transmitters move together, we estimate their similarity in $X$ or $Y$ coordinates using correlation coefficient. If the correlation coefficient for $X$ or $Y$ is over a certain threshold, we declare the transmitter pairs to be moving together. While it may be possible to combine the inference about the correlation in $X$ and the correlation in $Y$, we do not address this in this paper.

\section{Results}

\subsection{Experimental Methodology}

The measured environment is a typical office environment with partitioned cubicle offices. The experiments were performed during normal office hours where one could expect dynamic changes in the environment as a result of the mobility of the people within the office. We set up both IEEE 802.11b and IEEE 802.15.4 receivers within the office space and place them at strategic locations as shown by stars in Fig. 4. The WiFi receivers(landmarks) in these four locations operated in promiscuous mode in $2.4 \mathrm{GHz}$, ISM Band Channel 1 to capture all the packets in this particular channel. A Tmote Sky mote configured as receiver was attached to each of the landmarks to capture packets originating from Zigbee transmitters. These motes operated in $2.4 \mathrm{GHz}$, ISM Band Channel 16.

We used four IEEE 802.11b cards and four Tmote Sky motes as transmitters where a pair of $\mathrm{WiFi}$ cards and a pair of motes were placed together in the first laptop and the other pair of WiFi cards and motes were placed together in the second laptop as illustrated in Fig. 5. The motes were battery powered. The WiFi cards were connected to the configured APs and pinged the AP at the rate of 10 packets / sec with a transmit power of $15 \mathrm{dBm}$. And the motes were 


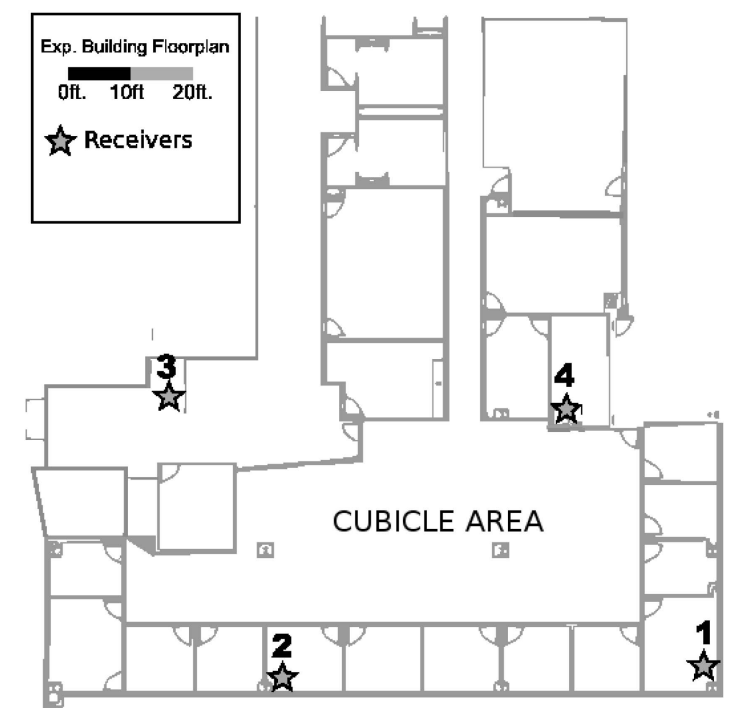

Fig. 4. Floorplan of the experiment environment and the node placement.

configured to transmit packets at the rate of 10 packets/sec at $0 \mathrm{dBm}$. We use the ORBIT infrastructure for capturing and logging each IEEE 802.11 and IEEE 802.15.4 packet from these transmitters to be stored in a SQL database. For each packet, we logged the transmitter's MAC address (Mote ID in case of motes), the receiver's MAC address (Mote ID in case of motes), RSSI and the time when the packet was captured. We also recorded the ground truth about which transmitter pairs were moving together along with the speed and the start and the end times of the different static and mobile periods of these transmitters manually. We note that we set up pairwise transmitters in our experiments to show how DECODE works, but our approach could be applied to a set of transmitters that are comoving.

Two experimenters carried one laptop each (containing two WiFi and two motes) and conducted the experiment. The total experiment lasted for 1 hour with alternating static and mobile periods as shown in Fig. 7. The authors were walking at $0.3 \mathrm{~m} / \mathrm{sec}(1 \mathrm{ft} / \mathrm{sec})$ for about 20 minutes. We call this experiment period Slow Mobility. We chose very slow speeds because this represents the most challenging case. The same experiment was repeated once more where the moving speed of the transmitters was increased from 0.3 to $1 \mathrm{~m} / \mathrm{sec}$ (normal human walking speed). We refer to this second experiment period as Walking Speed Mobility. We refer to these experiment traces as the complete traces.

To analyze the effect of mobility on the results, we then also create mobile-only traces by extracting and concatenating the two 10-minute mobile periods into a 20-minute mobile trace. Using this technique, we both create a slowmobile and a walk-speed mobile trace. We then use a timebased sliding window of time interval $T_{s}$ seconds to slice each of the above data sets into overlapping test traces. We vary the time interval $T_{s}$ from 10 to 400 seconds in steps of 10 seconds. For, example $T_{s}=100 \mathrm{~s}$ would generate 1,101 test traces of duration 100 seconds from the 1,200 seconds of data. We used these different sliced data sets with different time intervals $T_{s}$ in our results to report the detection rates and false positives.
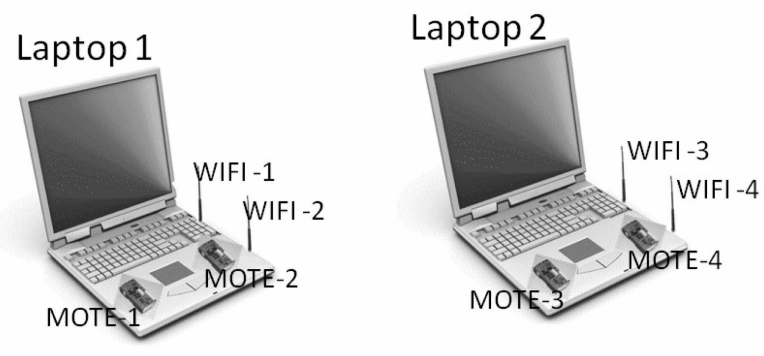

Fig. 5. Nodes and the transmitters used in experiments.

\subsection{Evaluation Metrics}

We will evaluate the effectiveness of RSS-based DECODE in the following three categories: 1) performance evaluation in terms of the detection rate of comoving transmitters and the corresponding false positive rate, 2) sensitivity study under different packet sampling rates and various correlation coefficient thresholds, and 3) generality investigation across different correlation methods and wireless networks. Finally, we will study the effects of mobility detection on the performance of DECODE.

\subsection{Effectiveness of DECODE's RSSI-Based Detector}

To evaluate the performance of DECODE, we first examine the detection rate and the false positive rate of determining the comobile transmitters. Fig. 6 depicts the detection rate and the false positive rate as a function of time with respect to each receiver for the IEEE 802.11 network for both Slow Mobility as well as Walking Speed Mobility experiments.

We compute the correlation coefficient for the samples accumulated over the last $T_{s}$ seconds and if the computed correlation coefficient is larger than 0.6, the pairs of transmitters are declared to be comobile. Otherwise, these pairs of transmitters are declared to be not moving together. A detailed discussion of the choice of the threshold is presented in Section 4.4. In our 20 minutes of mobile trace, we repeat the above procedure for all the generated data subsets of duration $T_{s}$ seconds. We then estimate detection rate as the percentage of times DECODE correctly reports comobility when the pairs of transmitters are indeed moving together and False positive rate as the percentage of times DECODE incorrectly reports comobility when the transmitters are not moving together.

Fig. 6 shows that in both the Walking Speed Mobility and Slow Mobility experiments, DECODE is able to detect all comoving and noncomoving pairs over all the data subsets accurately. We can also see that increasing the observation time $T_{s}$ improves the comobility detection rate while reducing the likelihood of observing spurious matches.

We found that the mobility speed also has an impact on the time required to achieve high detection rate and low false positive rate. In the Walking Speed Mobility experiment, it takes about 130 seconds to detect all comoving data subsets. Whereas it takes around 370 seconds to achieve the same in the Slow Mobility experiment. This suggests that with higher speed, more shadow fading effects can be observed within a shorter duration, leading to improved detection performance.

The results of the Slow Mobility experiment represent detection performance of DECODE under challenging 


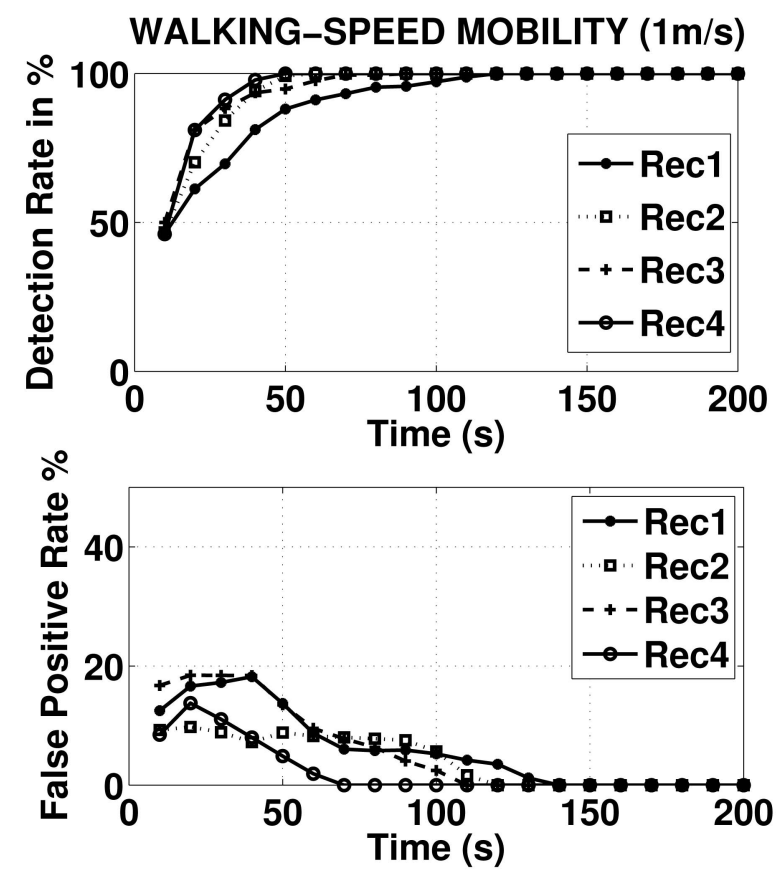

(a)
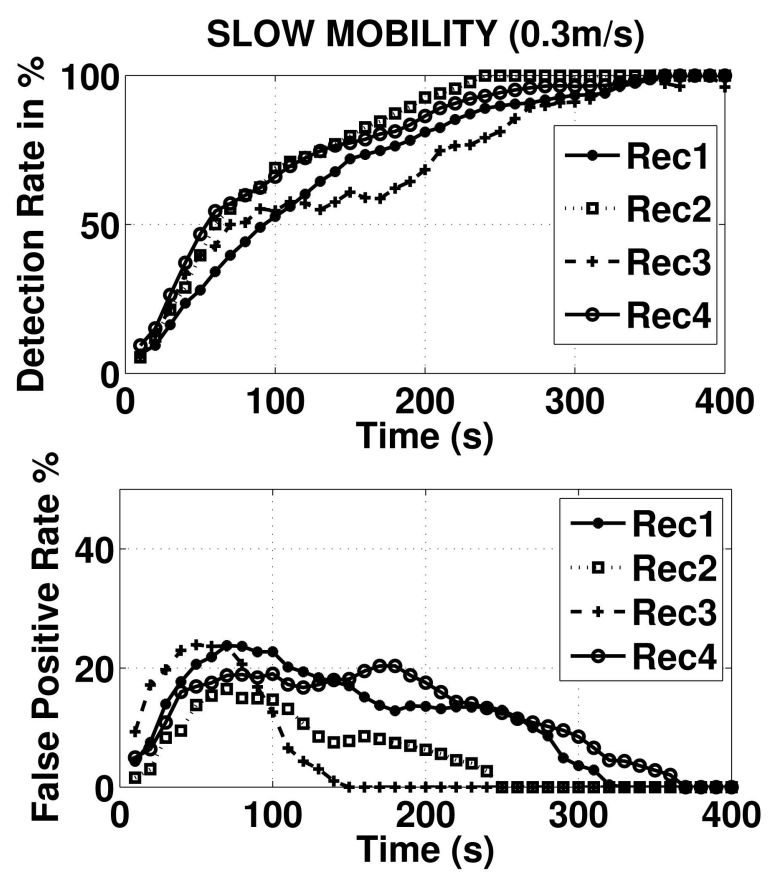

(b)

Fig. 6. IEEE 802.11 network: effectiveness of DECODE in terms of detection rate and false positive rate. Plots for the (a) Walking Speed Mobility experiment and (b) Slow Mobility experiment.

conditions. For the rest of this section, we provide analysis by using the Walking Speed Mobility experiment since it represents more typical scenarios for devices carried by humans.

\subsection{Sensitivity to Sampling Rate and Correlation Coefficient Threshold}

We now study the sensitivity of our scheme with respect to the different correlation coefficient thresholds and sampling rates, which we define to be the "packet transmission rate per transmitter." To this end, we further process the Walking Speed mobile trace and extract 0.5, 1, 5, and 10 packets every second from the trace to generate data sets corresponding to sampling rates of $0.5,1,5$, and 10 packets per second (pps), respectively. These four data sets are further sliced into several data subsets with time interval $T_{s}$ seconds similar to our previous study for estimating detection and false positive rates.

Fig. 8 presents the detection rate and false positive rate as a function of time for packet sampling rates of $0.5,1,5$, and 10 pps observed at receiver-2 (we do not present the results from other receivers as the performance is very similar). The threshold of the correlation coefficient is empirically determined to be 0.6 . We found that for the sampling rates of 1,5 , and $10 \mathrm{pps}$, the time taken to achieve 100 percent detection rate and 0 percent false positive rate is similar-about 130 seconds. With the low $0.5 \mathrm{pps}$, the time to reach 100 percent detection rate increases marginally to 150 seconds. This is encouraging as it indicates that

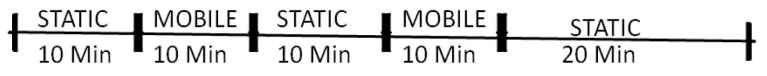

Fig. 7. The experimental procedure.
DECODE is not very sensitive to sampling rates in the 1 pps range. This insensitivity can be because a higher sampling rate would not provide additional gain compared to lower sampling rate as long as the lower sampling rate is fast enough to capture the "shadowing events."

This insensitivity to sampling rate also allows reducing the overall channel utilization, in a system design that relies on explicitly transmitted beacons to allow comovement detection. The transmission overhead would be negligible. For example, assuming a minimum packet length of 29 Bytes (28 Bytes of Frame and 1 Byte of Payload), an $802.11 \mathrm{~b}$ station transmitting one packet per second at $11 \mathrm{Mbits} / \mathrm{s}$ PHY rate takes $603.27 \mu \mathrm{sec}$ [26] which accounts only for 0.06 percent of channel utilization.

We next analyze the sensitivity of DECODE to the correlation coefficient thresholds $\tau$. Choosing an appropriate
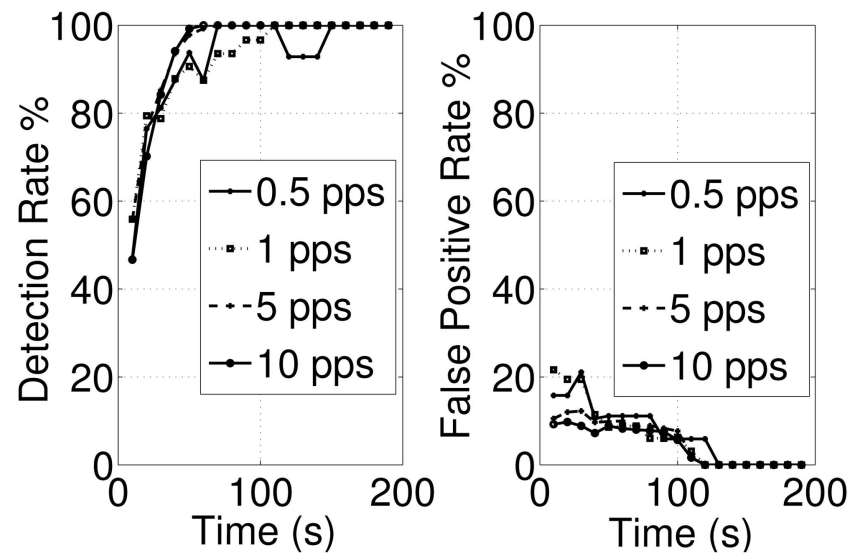

Fig. 8. IEEE 802.11 network: sensitivity of DECODE versus sampling rate. 

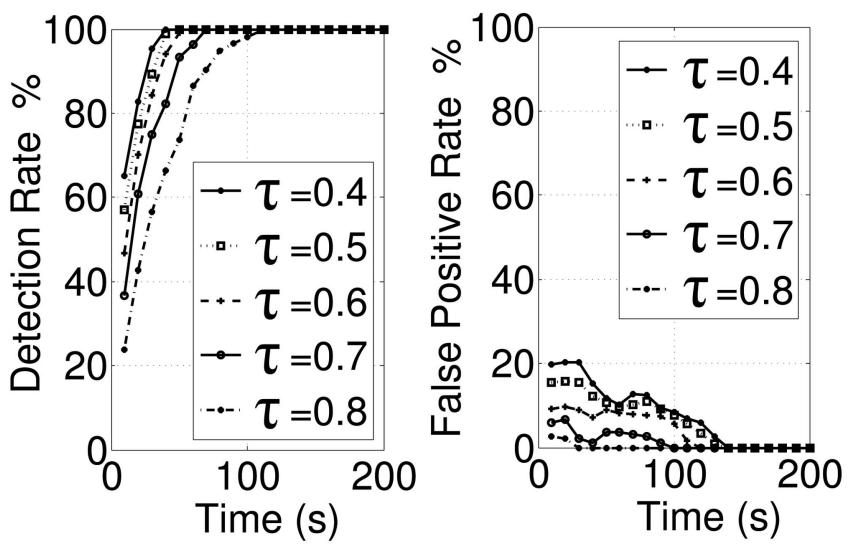

Fig. 9. Sensitivity of DECODE to correlation coefficient threshold. We pick a threshold of 0.6 for comovement.

threshold will allow our detection scheme to be robust to false detections. Fig. 9 presents the detection rate and the false positive rate for $\tau$ equaling $0.4,0.5,0.6,0.7$, and 0.8 . As expected, we observe that the detection rate takes longer to reach 100 percent as the threshold goes up, while the false positive rate drops to 0 percent quicker. The threshold $\tau=$ 0.6 achieves the best balance with a false positive rate remaining below 10 percent at all times and the detection rate reaching 100 percent nearly as fast as the smaller thresholds 0.4 and 0.5 . Hence, we chose a threshold of 0.6 for all other experiments.

\subsection{Generality of RSSI-Based DECODE}

We now study the generality of DECODE in using different correlation methods to determine comoving transmitters and its generality across both IEEE 802.11 as well as IEEE 802.15.4 networks.

Different correlation methods. We compare our correlation coefficient method (i.e., Pearson's product moment correlation coefficient) with Spearman's rank correlation coefficient in Figs. 10a and 10b for the IEEE 802.11 network and the IEEE 802.15.4 network, respectively. The correlation
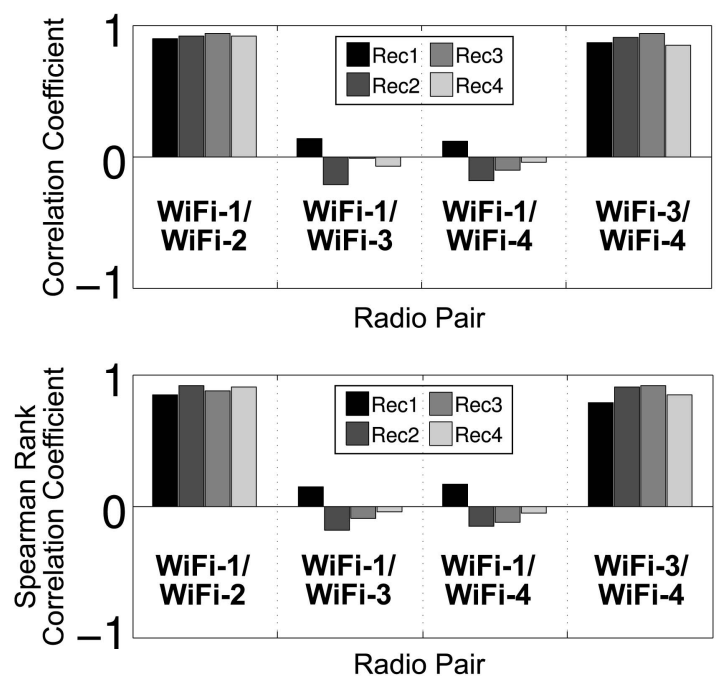

(a)

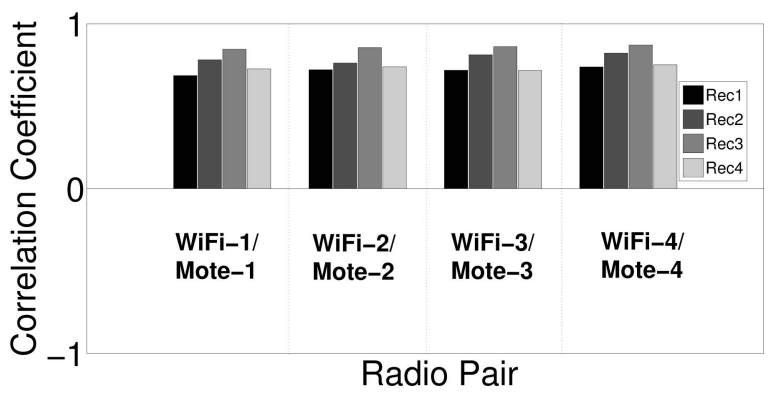

Fig. 11. Correlation coefficient for colocated Mote and WiFi.

coefficients are computed for all the comoving and noncomoving pairs of transmitters. Note that we refer to the Pearson's product moment correlation coefficient method as correlation coefficient in the figure.

We observed that both the correlation coefficient methods perform similarly for the comoving and the noncomoving pairs of transmitters. For the comoving pairs, the correlation coefficients from both methods are above 0.6 , while for the non-comoving pairs, both have values of correlation coefficient below 0.2 .

Different wireless networks. Fig. 11 presents the results of correlation coefficient calculated across an 802.11 transmitter and an 802.15.4 transmitter.

We found that the correlation coefficients for comoving pairs for both the 802.11 as well as for the 802.15.4 are consistently high (larger than 0.6 ) across all receivers. This is because when there is an obstruction to the Line-of-Sight signal component due to walls and other objects, both the WiFi and the mote transmitters experience similar shadowing effect as they are placed close enough. Though the actual amount of the degradation of signal differs, the relative effects are the same. Since Pearson's correlation coefficient method removes the sample mean from its estimation, similar relative performance is enough to capture comoving transmitters. This result is strong evidence that our approach is generic across different networks.
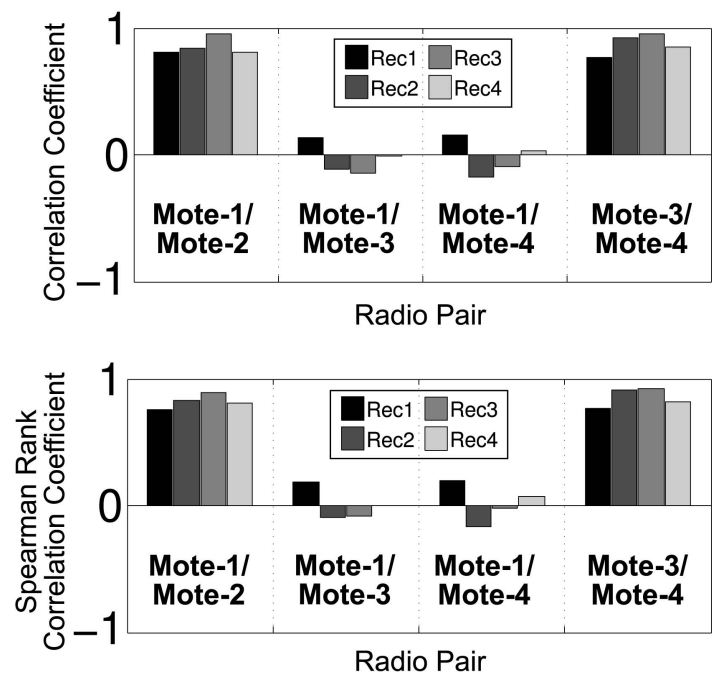

(b)

Fig. 10. Comparison of correlation coefficient methods for WiFi and Mote radio pairs. (a) IEEE 802.15.4 network and (b) IEEE 802.11 network. 


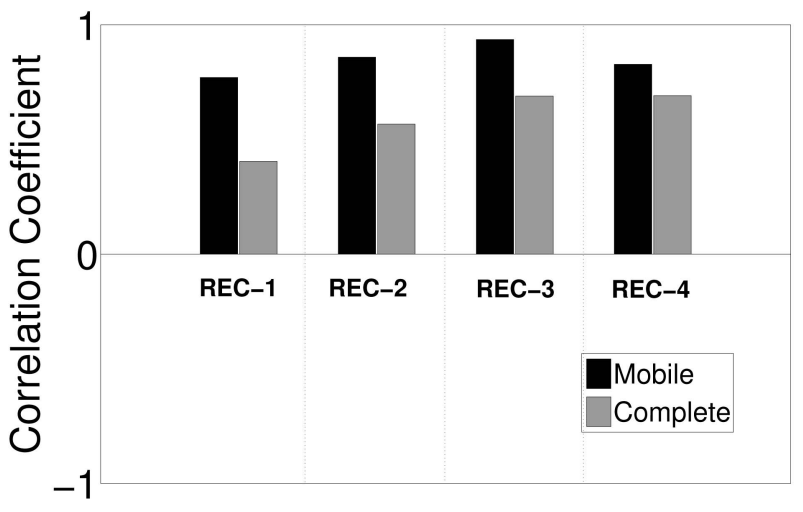

Fig. 12. 802.11 network: calculation of the correlation coefficient over the entire experimental period and over the mobile periods only. There is a 20 percent improvement in the correlation coefficient values when applied over mobile periods only.

\subsection{Significance of Mobility Detection for DECODE}

In this section, we examine how mobility detection impacts the performance of DECODE.

Effects of mobility detection. Fig. 12 plots the correlation coefficient at all four receivers for comoving transmitters in the Walking Speed Mobility experiment. The correlation coefficient is computed over the entire duration of the experiment as well as just over the mobile periods.

We found that the mobility detection helps in increasing the values of the correlation coefficient for comoving transmitters by an average of 20 percent. During static periods, the comoving transmitters do not experience significant changes in shadow fading, but may experience small scale fading effects that differ from one transmitter to the other (if the separation is more than about $6 \mathrm{~cm}(\lambda / 2)$ for $2.4 \mathrm{GHz}$ ). Thus, including static periods in the calculations tends to reduce overall detection performance, particularly if the static periods are long compared to the mobile periods.

These results support our approach of first extracting mobile (high variance) periods.

Thresholds for variance and window size. For mobility detection, there are several metrics available as shown in [8]. However, we found that using a simple metric, variance of RSS, is sufficiently effective. Further, two parameters are important when using the RSS variance to detect mobility: the threshold of variance and the number of RSS samples on
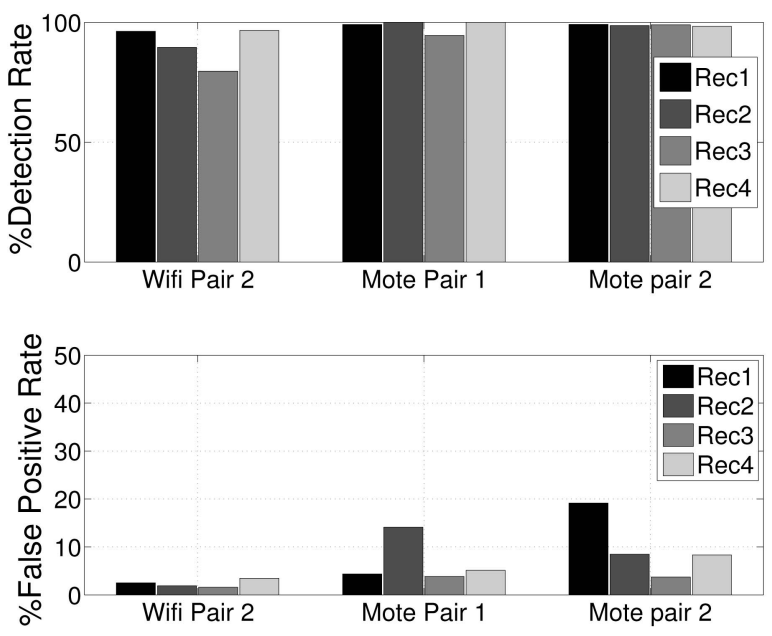

Fig. 14. Mobility detection of comoving transmitters under windowsize $=$ 50 ; variance threshold $=3$.

which the variance is calculated. Fig. 13 plots the trade-off between the detection rate and the false positive rate for different variance thresholds and different window sizes for the comoving WiFi transmitter pair.

We observed that the variance threshold of three has the highest detection rate with false positives less than 10 percent for all window sizes. We choose a window size of 50, where the detection rate is over 96 percent and the false positive is less than 1 percent. We estimate the correctness of these parameters across all transmitters to check the result consistency. Fig. 14 plots the detection rate and the false positive rate for mobility detection across the rest of the six transmitters including both WiFi and mote transmitters. The results from Fig. 14 prove that our results are consistent across all the transmitters with high detection rate and less than 10 percent false positive rate under a window size of 50 and a variance threshold of three.

\subsection{Comovement Detection in Location Space}

As pointed out in Section 2, the euclidean distance between the pairs of transmitters is not a very accurate estimator for comovement detection. In this section, we evaluate the DECODE correlation estimation applied to individual coordinates in location space.
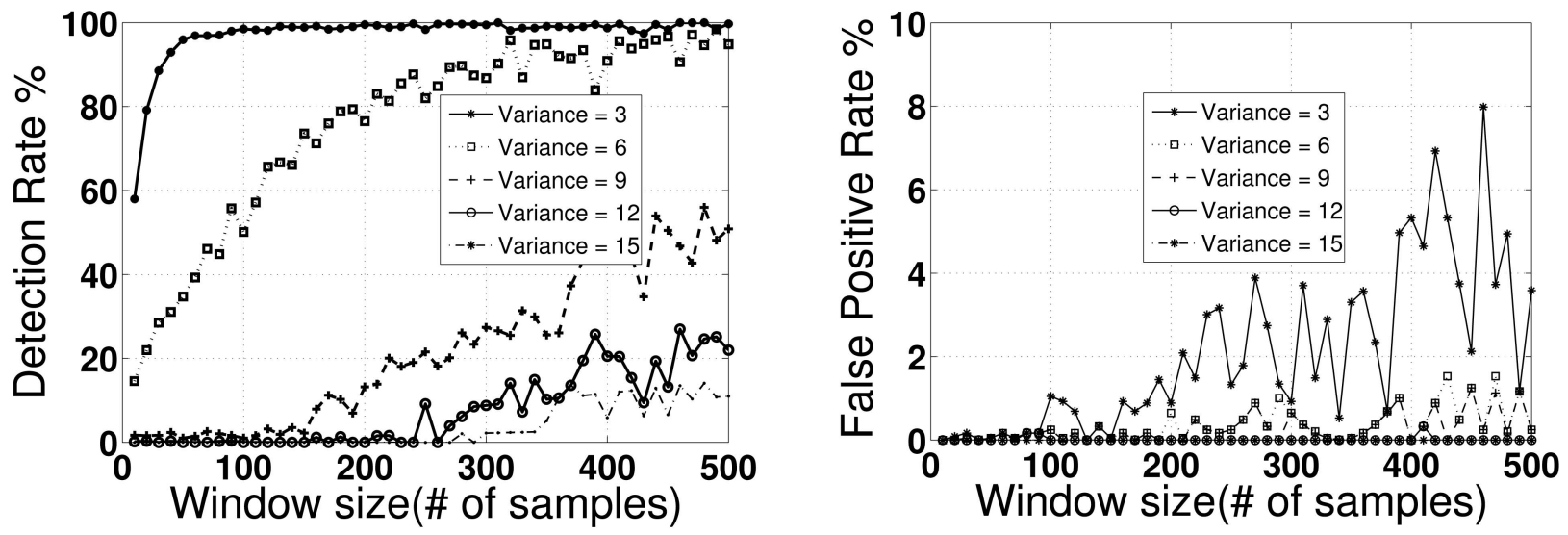

Fig. 13. Effects of variance threshold and sample window size on mobility detection. 

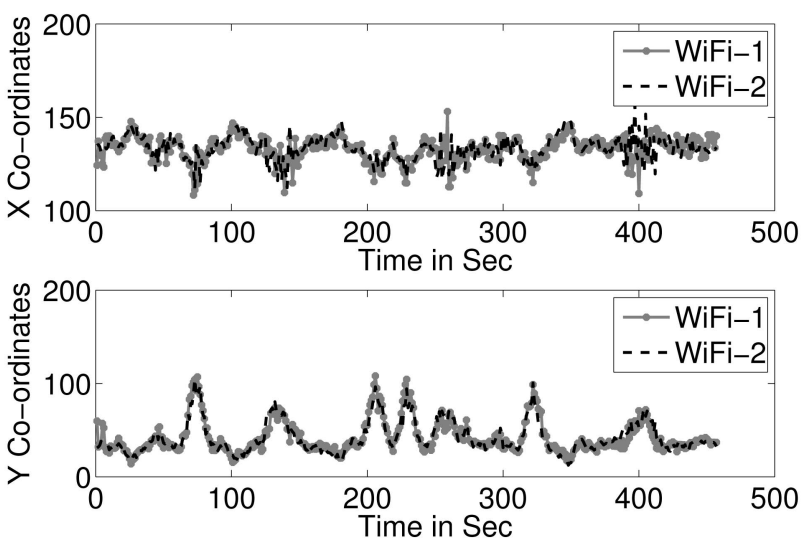

Fig. 15. Localized $X$ and $Y$ positions for a pair of comoving WiFi devices.

Fig. 15 plots the localized $X$ and $Y$ positions over time for a pair of comoving WiFi devices that were attached to the laptop 1 and moving at a speed of $1 \mathrm{~m} / \mathrm{sec}$. We can observe that the $X$ and $Y$ coordinates estimated by the localization system for the two comobile transmitters are very similar, but some differences exist. These differences can be attributed to the sensitivity of the localization algorithm to small scale fading, which can affect both transmitters differently and resulted in the high variance in euclidean distance, as was shown in Fig. 1.

However, by calculating the correlation coefficient over the localized $X$ position and the correlation coefficient over the localized $Y$ positions, we can achieve similar detection performance to the signal space technique. This is possible because the correlation coefficient can ignore the absolute values and can capture the relative trend in the way the $X$ and $Y$ coordinates vary (e.g, shadow fading is likely to lead to similar localization errors for both transmitters).

We evaluate the total time taken to achieve a 100 percent detection rate and 0 percent false positive rate. We define the detection rate as the percentage of times the correlation coefficient computed for a comoving pair is above 0.6 and false positive as the percentage of times the correlation coefficient for a noncomoving pair is above 0.6. Fig. 16 plots the detection rate and false positives as a function of time. Note that for simplicity, we have calculated correlation separately for the $X$ and $Y$ coordinates. We can see that it takes nearly 200 and 90 seconds for the $X$ and $Y$ coordinates, respectively, to achieve a 100 percent detection rate with 0 percent false positive rate. The corresponding time taken by DECODE in signal space was 130 seconds. While these times are comparable, there are several advantages of using signal space DECODE over location space DECODE-we discuss them in the Section 6.

\section{Simulation with Different Channel PARAMETERS}

After observing encouraging results in the experimental indoor environment, we evaluate now whether these experimental results as presented in Section 4 are consistent with results from simulation models and whether they can be generalized to indoor and outdoor environments with different propagation parameters. We also analyze the effect of shadow fading on the detection time.
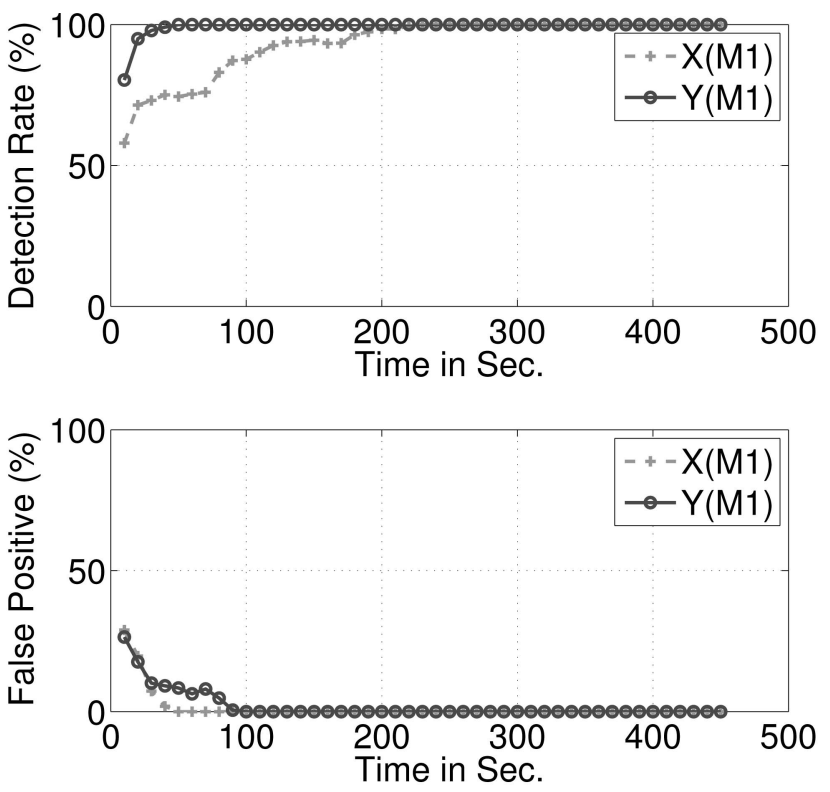

Fig. 16. Effectiveness of correlation coefficient applied over the localized $X$ and $Y$ locations for comovement detection.

Our simulation methodology involves generating the received power at a receiver from three transmitters, two of which are moving together on the same path and the third transmitter following a different path. To allow comparison with the experimental results, the path taken by the moving transmitters in the simulation was derived from the experiment paths described in Section 4.1.

The simulator generates received power levels as follows: From [27], we know that the received power at a receiver from a transmitter can be modeled as:

$$
P(d)=P_{0}-10 \gamma \log _{10}\left(\frac{d}{d_{0}}\right)+S_{\sigma}+\delta,
$$

where $d$ is the distance between the transmitter and the receiver; $P_{0}$ is the received power at the reference distance $d_{0}$ from the transmitter; $\gamma$ is the path loss exponent; $S_{\sigma}$ represents shadow fading (i.e., correlated shadowing) which follows zero mean and $\sigma$ standard deviation Gaussian distribution and $\delta$ is the random noise.

To simulate correlated shadowing, the $S_{\sigma}$ for different positions must satisfy the following exponential constraint [28]:

$$
E\left[S_{\sigma}\left(P_{i}\right) S_{\sigma}\left(P_{j}\right)\right]=\sigma^{2} e^{-D_{i j} / D_{c}},
$$

where $S_{\sigma}\left(P_{i}\right)$ and $S_{\sigma}\left(P_{j}\right)$ are the shadow fading at location $P_{i}$ and $P_{j}$, respectively. $D_{i j}$ is the distance between the positions $P_{i}$ and $P_{j} . D_{c}$ represents the decorrelation distance, which can range from 1-2 $\mathrm{m}$ indoors to many tens of meters outdoors. We generate such correlated Gaussian random variables $S_{\sigma}$ by multiplying uncorrelated Gaussian random variables with the upper triangular matrix from a Cholesky decomposition of the correlation matrix [29]. In our case, the correlation matrix is initialized with the desired correlation values $e^{-D_{i j} / D_{c}}$ between each transmitter (position) pair.

As shown in Table 1, we considered four scenarios with different propagation parameters, two for indoor environments and two for outdoor environments. For the indoor 
TABLE 1

Summary of the Parameters Used in Simulations along with the Total Detection Time

\begin{tabular}{|c|c|c|c|c|c|}
\hline Environment & PathLoss Exponent & De-Correlation Distance (m) & $\sigma_{\text {CorrelatedShadowing }(\mathrm{db})}$ & $\sigma_{N o i s e}(d b)$ & Detection Time (sec) \\
\hline Indoor-1 & 2.5 & 2 & 2 & 2.3 & 108 \\
\hline Indoor-2 & 2.5 & 2 & 4.3 & 0 & 81 \\
\hline Outdoor-1 & 2.8 & 5 & 2 & 2.4 & 94 \\
\hline Outdoor-2 & 2.8 & 5 & 4.4 & 0 & 70 \\
\hline
\end{tabular}

environments, we chose standard deviation of the received power by measuring in our experiment environment. Since this standard deviation combines both correlated shadowing and random noise, we simulate two indoor scenarios with different assumptions on the level of shadowing and noise. While Gudmundson's exponential [28] decay model has been proposed for medium to large cellular networks in the outdoor environments, Jalden et al. [7] have shown that this exponential model can be adapted for analyzing the spatial correlation arising from shadowing in the indoor environments. We obtained the other indoor and outdoor parameters including the propagation exponent from other reported measurements [7], [30]. The last column of the table also shows the result, the total time taken for detecting comovement without false positives. The results show similar detection times across all four scenarios, indicating that DECODE is not very sensitive to propagation parameters. This is encouraging and shows that DECODE can be expected to also work in outdoor environments with typical parameter settings.

While the simulation results show slightly lower detection times, 80-108 seconds compared to 130 seconds in the experiment, the results are on the same order of magnitude. The difference can be attributed to modeling and measurement inaccuracies. We measured the standard deviation in power $(4.3 \mathrm{~dB})$ within the office environment several months after conducting the DECODE experiments. Also, the simulation model assumes that power measurements follow a Gaussian distribution $\mathcal{N}\left(P_{0}-10 \gamma \log _{10}\left(d_{n} / d_{0}\right), \sigma_{R S S}^{2}\right)$, which may not be fully accurate.

The indoor results also show that increasing the correlated shadowing reduces the overall detection time from 110 seconds to 81 seconds. A similar trend can be observed in the outdoor results. This indicates that the presence of correlated shadow fading leads to faster detection and is beneficial for DECODE.

\section{Discussion}

In this section, we discuss the feasibility of detecting transmitters that are static and located within close proximity. We continue the discussion by giving out the advantages of operating in the signal space in comparison to the location space. We finally conclude this section by discussing the impact of missing samples on comobility detection.

\subsection{Feasibility of Detecting Colocation}

The comovement detection results described so far raise the question whether the DECODE technique can also be used to detect stationary colocated transmitters. Ostensibly, an environment with high surrounding mobility could lead to similarly high signal variance even though the transmitters and receivers are stationary, because the moving objects can temporarily block transmission paths, which changes shadow and multipath fading patterns.

To investigate whether human mobility in a cubicle office environment is sufficient to also allow detection of colocated stationary transmitters, we performed an experiment where a pair of mote transmitters were attached to the main doorway within the WINLAB office, which is an area with frequent human traffic (it is located next to a printer and water cooler providing additional traffic).

Table 2 shows the correlation coefficients obtained for the stationary transmitter pairs by each of the receivers over a 2,000-second interval (the transmitters actually moved when the door was opened, but this occurred only twice in this period). Note that all correlation coefficients are far below the 0.6, our correlation threshold for comovement detection. Note also that some of the receivers show correlation coefficient values near zero, which suggests that reducing the detection threshold would not be very effective. Thus, these results show that in a typical office environment, surrounding mobility is unlikely to induce sufficient correlated fading to allow use of the DECODE technique for detecting colocated transmitters (even with the extended 2,000-second measurement period, compared to the 130-second period that was sufficient for comovement detection as shown previously).

\subsection{RSSI-Based versus Location-Based Detection}

While accuracy of DECODE in both signal and locationspace is similar, applying DECODE in signal-space provides several advantages, particularly if location information is not needed for other applications. However, there are challenges to be addressed before one could assume localization systems are sufficient for the purposes of colocation detection:

- Generality: Most localization systems use the already computed training set to determine the location associated with any fingerprint. However, this approach requires the TX power settings during the training and the testing phase to be same in order to estimate the correct location. With a wide variety of wireless devices in the environment, this requirement makes localization technique highly sensitive and error prone, while RSS-based comovement detection is more agnostic to these issues. Also, different radio technologies may need different

TABLE 2

Correlation Coefficient for the

Time Interval $\mathrm{t}=3,100$ seconds to $\mathrm{t}=5,100$ seconds

\begin{tabular}{|c|c|c|c|}
\hline Receiver1 & Receiver 2 & Receiver 3 & Receiver 4 \\
\hline 0.4630 & -0.1140 & 0.2753 & 0.4362 \\
\hline
\end{tabular}




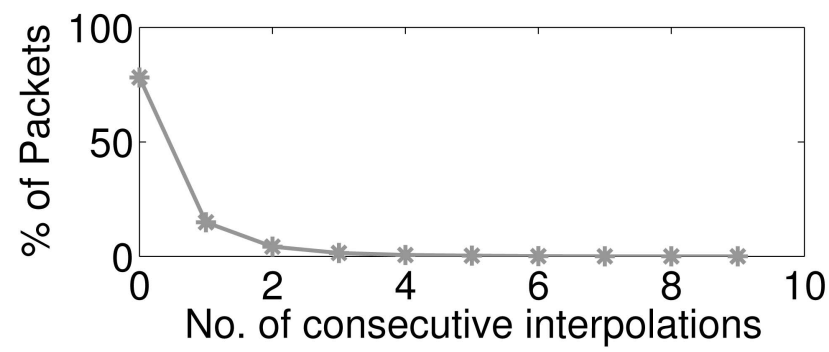

Fig. 17. Histogram of the percentage of consecutive missing samples from a WiFi transmitter observed at Receiver-1.

localization systems with different accuracy limits, making colocation detection for radios belonging to different technologies nontrivial using these systems, while we showed that the RSS-based technique can be used across wireless technologies.

- Localization Overheads: Calculating absolute location of a device takes time and requires signal information from multiple points of contact (e.g., three reference points for trilateration), which may not be available at all times.

- Infrastructure Costs: Investing in the localization infrastructure, including the equipment costs as well as maintaining signal maps, beacon or landmark (receiver) positions, etc., might be costly. In comparison, the RSS-based comovement detection techniques only requires a single receiver.

\subsection{Impact of Missing Samples on Comobility Detection}

As explained in Section 3.1, the time alignment step involves replacing the missing samples from a transmitter with its last observed sample. While this step aids in comparison of signal strengths from transmitter pairs, excessive replacement of missing samples could overstate the correlation between transmitter pairs. To this end, we analyze the percentage of times the missing samples have been replaced during this step for a WiFi-WiFi pair at Receiver-1.

Fig. 17 shows that very few consecutive packet losses occur. Eighty percent of packets have been correctly received. Fifteen percent are intermittent single packet losses and only 5 percent of packets are consecutive packet losses. We also verified these packet loss rates across all the other transmitter receiver pairs and found similar results. Since DECODE uses a moving window average over 1 second of received packets to remove multipath fading, interpolation of these packet losses has little effect on the correlation results.

\section{ConClusion}

In this work, we presented DECODE, a system that detects comoving wireless devices. DECODE's strategy is founded on exploiting the similarity in shadow fading for the packets transmitted from a set of transmitters. We showed that our technique can work in both the signal space and its corresponding location space, but that the signal space approach provides the key advantage that only a single base station is needed. Our approach was general in that it could detect comovement of wireless transmitters with different radios. We validated our approach through simulations for various indoor and outdoor environments. We further demonstrated that it works for both IEEE 802.11b/g WLAN and IEEE 802.15.4 Mote devices in real indoor environments.

Given 130 seconds of mobile data, DECODE can achieve a true positive rate of 100 percent with 0 percent false positive estimated over 1,071 data subsets. However, a key finding of this work is that mobility is critical for our approach, and that the DECODE's effectiveness scales with both the time and speed of the devices mobility. We also showed that DECODE's performance is insensitive to the sampling rate and a sampling rate of 1 packet/second for 130 seconds was sufficient to achieve a 100 percent detection rate and 0 percent false positive rate.

Finally, because DECODE's effectiveness is quite sensitive to mobility, we examined using the RSSI variance for mobility detection. We found this technique to have a mobility detection rate of over 96 percent with the corresponding false positives to be less than 1 percent. Therefore, detecting mobility has a straightforward solution and does not limit the DECODE system.

\section{ACKNOWLEDGMENTS}

This material is based upon work supported by the US National Science Foundation under Grant No. CNS 0627032. This work is supported in part by the US Army, Picatinny, under Contract No. W15QKN-05-D-0011.

\section{REFERENCES}

[1] E. Elnahrawy, X. Li, and R.P. Martin, "The Limits of Localization Using Signal Strength: A Comparative Study," Proc. First IEEE Int'l Conf. Sensor and Adhoc Comm. and Networks (SECON), pp. 406414, Oct. 2004.

[2] Y. Cui and S.S. Ge, "Autonomous Vehicle Positioning with GPS in Urban Canyon Environments," IEEE Trans. Robotics and Automation, vol. 19, no. 1, pp. 15-25, Feb. 2003.

[3] N. Eagle and A.S. Pentland, "Reality Mining: Sensing Complex Social Systems," Personal Ubiquitous Computing, vol. 10, no. 4, pp. 255-268, 2006.

[4] Z. Rong and T.S. Rappaport, Wireless Communications: Principles and Practice. Prentice Hall, 2002.

[5] C. Hill and T. Kneisel, "Portable Radio Antenna Performance in the 150, 450, 800, and $900 \mathrm{MHz}$ Bands Outside and In-Vehicle," IEEE Trans. Vehicular Technology, vol. 40, no. 4, pp. 750-756, Nov. 1991.

[6] P. Nepa, G. Manara, S. Mugnaini, G. Tribellini, S. Cioci, G. Albasini, and E. Sacchi, "Differential Planar Antennas for 2.4/5.2 GHz WLAN Applications" Proc. IEEE Int'l Symp. Antennas and Propagation Soc., pp. 973-976, July 2006.

[7] N. Jalden, P. Zetterberg, B. Ottersten, A. Hong, and R. Thoma, "Correlation Properties of Large Scale Fading Based on Indoor Measurements," Proc. IEEE Wireless Comm. and Networking Conf. (WCNC '07), Mar. 2007.

[8] T. Sohn, A. Varshavsky, A. LaMarca, M.Y. Chen, T. Choudhury, I. Smith, S. Consolvo, J. Hightower, W.G. Griswold, and E. de Lara, "Mobility Detection Using Everyday GSM Traces" Proc. Int'l Conf. Ubiquitous Computing (UbiComp '06), pp. 212-224, Sept. 2006.

[9] K. Muthukrishnan, M. Lijding, N. Meratnia, and P. Havinga, "Sensing Motion Using Spectral and Spatial Analysis of WLAN RSSI," Proc. Ninth Workshop Mobile Computing Systems and Applications (EuroSSC '07), Oct. 2007.

[10] J. Krumm and E. Horvitz, "Locadio: Inferring Motion and Location from wi-fi Signal Strengths" Proc. Int'l Conf. Mobile and Ubiquitous Systems: Networking and Services (MOBIQUITOUS '04), pp. 4-13, Aug. 2004.

[11] N. Eagle and A. Pentland, "Social Serendipity: Mobilizing Social Software," IEEE Pervasive Computing, vol. 4, no. 2, pp. 28-34, Apr./ June 2005. 
[12] J. Hightower, R. Want, and G. Borriello, "SpotON: An Indoor 3D Location Sensing Technology Based on RF Signal Strength," Technical Report UW CSE 2000-02-02, Univ. of Washington, Dept. of Computer Science, Feb. 2000.

[13] R. Want, A. Hopper, V. Falcao, and J. Gibbons, "The Active Badge Location System," ACM Trans. Information Systems, vol. 10, no. 1, pp. 91-102, Jan. 1992.

[14] N. Priyantha, A. Chakraborty, and H. Balakrishnan, "The Cricket Location-Support System" Proc. ACM MobiCom, pp. 32-43, Aug. 2000.

[15] A. Krishnakumar and P. Krishnan, "On the Accuracy of Signal Strength-Based Location Estimation Techniques," Proc. IEEE INFOCOM, Mar. 2005.

[16] P. Bahl and V. Padmanabhan, "Radar: An In-Building rf-Based User Location and Tracking System," Proc. IEEE INFOCOM, vol. 2, pp. 775-784, 2000.

[17] Y. Chen, J. Francisco, W. Trappe, and R.P. Martin, "A Practical Approach to Landmark Deployment for Indoor Localization," Proc. Third Ann. IEEE Comm. Soc. Conf. Sensor, Mesh and Ad Hoc Comm. and Networks (SECON '06), Sept. 2006.

[18] A.M. Ladd, K.E. Bekris, A. Rudys, G. Marceau, L.E. Kavraki, and D.S. Wallach, "Robotics-Based Location Sensing Using Wireless Ethernet," Proc. ACM MobiCom, pp. 227-238, 2002.

[19] Y. Chen, G. Chandrasekaran, E. Elnahrawy, J.-A. Francisco, K. Kleisouris, X. Li, R.P. Martin, R.S. Moore, and B. Turgut, "Grail: General Real Time Adaptable Indoor Localization," Sensor Rev., 2008.

[20] M. Maróti, P. Völgyesi, S. Dóra, B. Kusý, A. Nádas, A. Lédeczi, G. Balogh, and K. Molnár, "Radio Interferometric Geolocation," Proc. Third Int'l Conf. Embedded Networked Sensor Systems (SenSys '05), pp. 1-12, 2005.

[21] B. Kusy, A. Ledeczi, and X. Koutsoukos, "Tracking Mobile Nodes Using rf Doppler Shifts," Proc. Fifth Int'l Conf. Embedded Networked Sensor Systems (SenSys '07), pp. 29-42, 2007.

[22] K.G. Calkins, E-Book, An Introduction to Statistics, http:// tinyurl.com/2jlrsu, 2008 .

[23] MathWorld, Spearman's Rank Correlation Co-Efficient, http:// tinyurl.com/3ymsre, 2007.

[24] K. Srinivasan and P. Levis, "RSSI is Under Appreciated," Proc. Third Workshop Embedded Networked Sensors (EmNets '06), May 2006.

[25] N. Patwari and S.K. Kasera, "Robust Location Distinction Using Temporal Link Signatures," Proc. ACM MobiCom, Sept. 2007.

[26] Cisco, Inc., Maximum Throughput Calculations for $802.11 \mathrm{~b}$ WLAN, http://tinyurl.com/kv3yjn, 2008.

[27] V. Erceg, L.J. Greenstein, S.Y. Tjandra, S.R. Parkoff, A. Gupta, B. Kulic, A.A. Julius, and R. Bianchi, "An Empirically Based Path Loss Model for Wireless Channels in Suburban Environments," IEEE J. Selected Areas in Comm., vol. 17, no. 7, pp. 1205-1211, July 1999.

[28] M. Gudmundson, "Correlation Model for Shadow Fading in Mobile Radio Systems," Electronics Letters, vol. 27, pp. 2145-2146, 1991.

[29] J.H. Stapleton, Linear Statistical Models. Wiley-Interscience, 1995.

[30] X. Zhao, J. Kivinen, P. Vainikainen, and K. Skog, "Propagation Characteristics for Wideband Outdoor Mobile Communications at 5.3 GHz," Proc. IEEE J. Selected Areas in Comm., Apr. 2002.

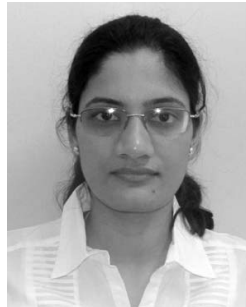

Gayathri Chandrasekaran received the MSc (Tech) degree in information systems from BITS Pilani, India. She is currently working toward the $\mathrm{PhD}$ degree in the Department of Computer Science at Rutgers University and is a research assistant in the Wireless Information Networking Laboratory (WNLAB) since 2006. Her research interests include pervasive computing, wireless device localization, and experimental evaluation of networking protocols. She is a recipient of the University Fellowship at the Ohio State University between the years 2004-2006 and the JNCASR summer research fellowship in the years 2003 and 2004.

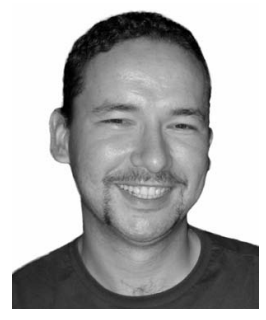

Mesut Ali Ergin received the BS degree in control and computer engineering from Istanbul Technical University, Turkey, in 1999 and the MS degree in computer engineering from Bogazici University, Turkey, in 2003. He is currently working toward the $\mathrm{PhD}$ degree in the Department of Electrical and Computer Engineering at Rutgers, the State University of New Jersey, and is a graduate assistant in the Wireless Information Networks Laboratory (WINLAB) since 2004. His research focus is on the diverse aspects of wireless and mobile networking systems.

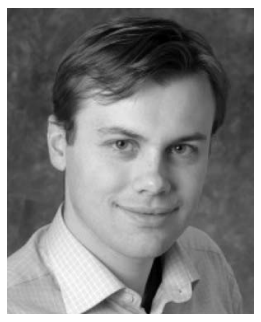

Marco Gruteser received the PhD degree in computer science from the University of Colorado at Boulder. He is currently an assistant professor at the Wireless Information Networks Laboratory (WINLAB), Rutgers University. His research interests include location-aware networking and building reliable, secure, and privacy-aware communication systems for vehicular networks. He is a member of the IEEE Computer Society and the ACM.

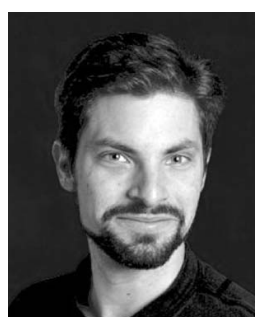

Richard P. Martin received the BA degree from Rutgers University and the MS and PhD degrees in computer science from the University of California at Berkeley. He is an associate professor of computer science at Rutgers University. He is a member of the Wireless Network Information Laboratory (WINLAB). His current research interests include wireless device localization and human factors in dependable computing. His awards include the Best Paper Award at the 2004 IEEE Conference on Sensor and Ad Hoc Communication Networks as well as a CAREER award from the US National Science Foundation (NSF). He has served as an investigator on grants from the Defense Advanced Research Projects Agency, the US National Science Foundation, and IBM.

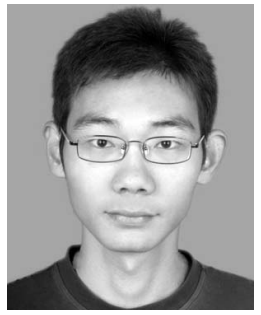

Jie Yang received the $\mathrm{BE}$ degree in automatic control from Beijing Institute of Technology, China, in 2004. He was in the PhD program in the Department of Automatic Control of Beijing Institute of Technology from 2005 to 2007 . He is currently working toward the $\mathrm{PhD}$ degree in the Electrical and Computer Engineering Department at Stevens Institute of Technology. His research interests include the areas of information security and privacy, wireless localization, and location-based services (LBS).

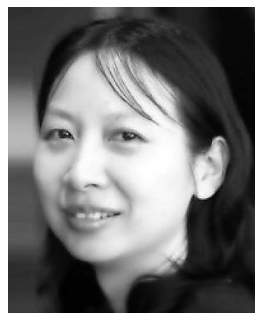

Yingying Chen received the PhD degree in computer science from Rutgers University. She is currently an assistant professor in the Department of Electrical and Computer Engineering at Stevens Institute of Technology. Her research interests include wireless and system security, wireless networking, and distributed systems. She is the coauthor of the book Securing Emerging Wireless Systems (Springer, 2009). Prior to joining Stevens Institute of Technology, she was with Bell Laboratories and the Optical Networking Group, Lucent Technologies. She received the IEEE Outstanding Contribution Award from the IEEE New Jersey Coast Section each year from 2000 to 2005. She was the recipient of the Best Technological Innovation Award from the Third International TinyOS Technology Exchange in 2006. 